\title{
Newborn English Bulldogs with Multiple Malformation Syndrome and Lethal Characters
}

\author{
Si-Jung Jang ${ }^{1, \#}$, Minkyung Kim ${ }^{1, \#}$, Hyeon-Jeong Lee ${ }^{1, \#}$, Young-Sung You ${ }^{2}$, Jaehoon Lee ${ }^{1}$ and \\ Sung-Lim Lee Le $^{1,3, *}$ \\ ${ }^{1}$ College of Veterinary Medicine, Gyeongsang National University, Jinju 52828, Korea \\ ${ }^{2}$ Helix Animal Medical Center, Goyang 412220, Korea \\ ${ }^{3}$ Research Institute of Life Sciences, Gyeongsang National University, Jinju 52828, Korea
}

Received June 13, 2019

Revised August 26, 2019

Accepted September 11, 2019

*Correspondence

Sung-Lim Lee

E-mail: sllee@gnu.ac.kr

ORCID

https://orcid.org/0000-0002-1055-8097

"These first authors equally contributed to this work.

\begin{abstract}
A female English bulldog was gave birth two neonates by cesarean section on the sixty one days after mating, but both neonates were died soon after birth. The bodies of neonates were diagnosed using radiography, ultrasonography, computed tomography and necropsy immediately after death. Both neonates had caudal regression syndrome, butterfly vertebra, hydrocephalus, umbilical hernia, cleft palate and bow-legged hind-limb. At necropsy, neonates had mild fetal anasarca, cleft lip and the skull was remained non-union. At thoracic cavity, only three ribs and thoracic spines were existed and patent ductus arteriosus was found. At abnormal cavity, the renal ectopia was found with abnormal morphology. In the present case, those English bulldog neonates with multiple congenital malformation syndromes seriously suffered vertebral column anomalies and that may induced by neural tube defects in during embryonic period. To prevent congenital malformation occurring in English bulldog, further in depth studies are needed for the breed specific genetic diversity and for the reason of behind genetic abnormality in these breed.
\end{abstract}

Keywords: caudal regression syndrome, congenital malformation, dog, English bulldog, teratology

\section{INTRODUCTION}

Dogs are genetically at high risk for congenital malformations, and genetic traits between breeds are known to increase due to not only the malformations of the generators but also the incidence of certain diseases. The causes of neural tube defects (NTDs) have not been elucidated clearly, but it has been reported to be caused by genetic, nutritional, and environmental factors or combination of these (Au and Ashley-Koch, 2010; Hertzler et al., 2010). The formation of neurons is developed by primary neurulation and secondary neurulation and in particular, the brain and most of the spinal cord are formed by primary neurulation. In case of the failure in closure or development of the neural tube caused by defect of primary neurulation, spina bifida which is an open form of NTDs can be led to happen (Bassuk and Kibar, 2009). The spina bifida causes various physical and developmental abnormalities depending on the magnitude and position of the spinal defect (Bassuk and Kibar, 2009). Harris et al. have reported to verify over 200 genes related to the spina bifida in the mouse model, but studies of direct genetic causes are rarely reported (Harris, 2009; Harris and Juriloff, 2010). And especially, there have been no reports to 
identify the cause of NTDs in dogs.

The caudal regression syndrome is a rare congenital malformation ranging from simple anal atresia to absence of sacral, lumbar and posterior thoracic vertebrae (Sen and Patel, 2007). Maternal diabetes, vascular hypoperfusion and genetic predisposition have been suggested as possible causative factors (Aslan et al., 2001). However, the caudal regression syndrome is rarely reported in human, and there are no cases have ever been reported academically in dogs.

In this report, we described two English bulldog fetuses delivered with multiple malformation syndrome including spina bifida and caudal regression syndrome, two rarely reported congenital malformations in dogs.

\section{CASE REPORT}

A 21-month-dog, $20 \mathrm{~kg}$, intact female, English bulldog was presented for parturition after 60 days from mating. The patient has a history of abortion in six-month ago after showing swelling of the mammary gland and abdomen after mating with the same male. A complete blood count $(\mathrm{CBC})$ and serum biochemical analysis of the patient was not shown significant change except mild de- crease in packed cell volume (PCV). Radiography revealed the presence of two offspring (Fetus 1; F1 fetus, Fetus 2; F2 fetus) with showing cranial bone in left abdomen but spinal bone was not confirmed clearly in both fetuses (Fig. $1 \mathrm{~A}$ and $\mathrm{B})$. On abdominal ultrasonographic examination, the F1 fetus was shown $66+/-3$ days of gestational age, $244 / \mathrm{min}$ of heart rate, and $2.5 \mathrm{~mm}$ of anasarca evaluation. The F2 fetus was also shown $66+/-3$ days of gestational age, $234 / \mathrm{min}$ of heart rate, and $4.3 \mathrm{~mm}$ of anasarca evaluation with possibility of fetal anasarca (Fig. 1C and D). Both fetuses were found to predisposition of hydrocephalus and it was confirmed that both fetuses have no vertebrae.

To determine cesarean section point, the rectal temperature and the fetal heartbeat were monitored. After 1 day from hospitalization, the cesarean section was performed by confirming the decrease of the rectal temperature (from $38.9^{\circ} \mathrm{C}$ to $37.9^{\circ} \mathrm{C}$ ) and the fetal heartbeat (from $234-244 /$ min to $200 \pm 10 / \mathrm{min})$. The patient was premedicated with cefazolin $(25 \mathrm{mg} / \mathrm{kg}, \mathrm{IV})$ and tramadol $(2 \mathrm{mg} / \mathrm{kg}, \mathrm{IV})$. Anesthesia induction was performed with propofol $(6 \mathrm{mg} /$ $\mathrm{kg}, \mathrm{IV})$. Anesthesia was maintained using isoflurane, and local anesthesia was performed with lidocaine and bupivacaine $(1 \mathrm{ml} / \mathrm{kg}$, IM). Cesarean section operation was
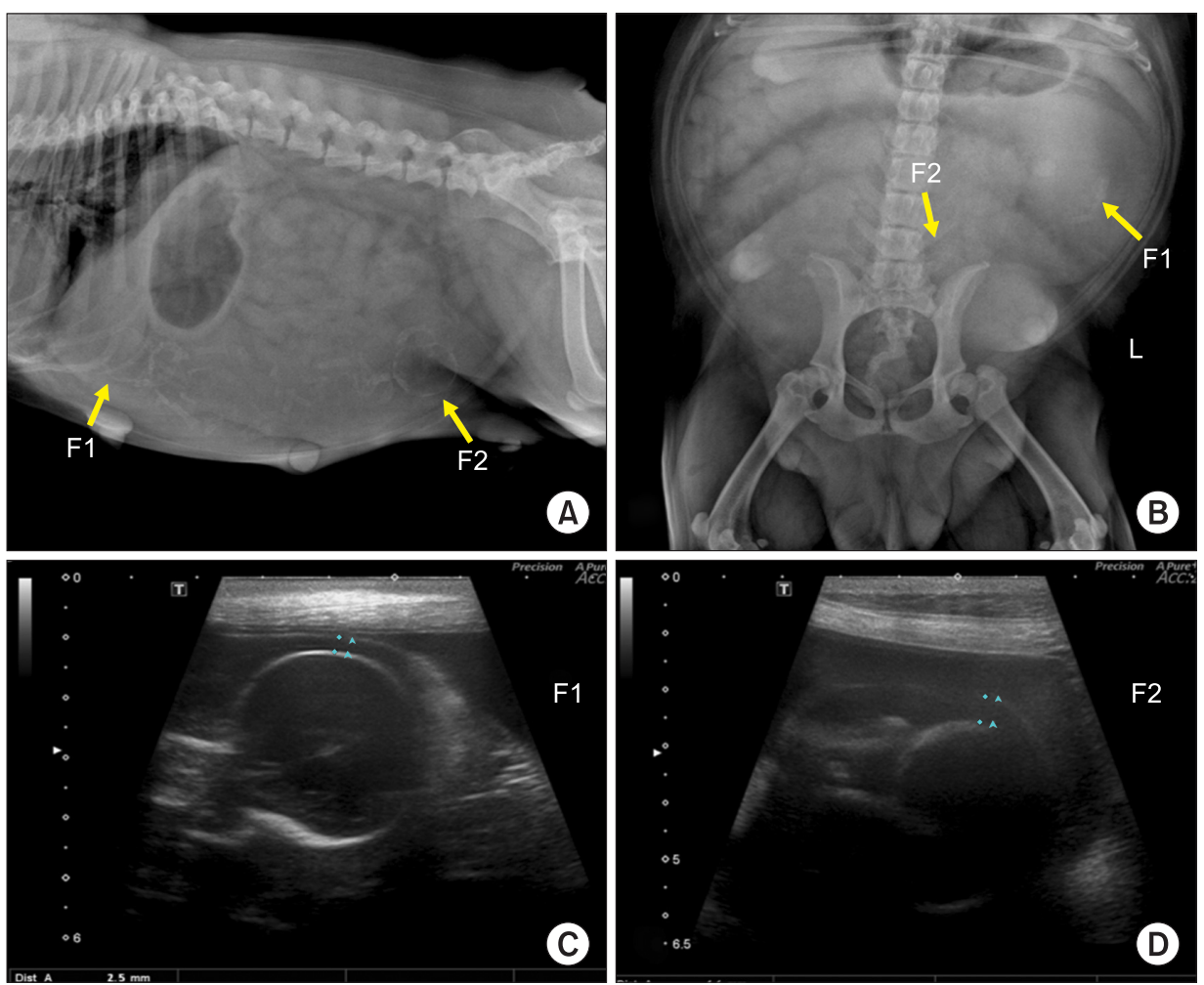

Fig. 1. Fetuses images of radiography and ultrasonography. (A, B) Two fetuses were presented in cranial (F1) and caudal (F2) part of abdomen by both left lateral (A) and ventrodorsal (B) radiohraphs. (C, D) Ultrasonogram of both Fetus 1 (C) and Fetus 2 (D) at preoperation. F1, fetus 1; F2, fetus 2 . 
performed following common procedure.

Two pups were delivered, but both were died immediately after the cesarean section. Both fetuses were observed caudal regression syndrome, butterfly vertebrae, hydrocephalus, umbilical hernia, cleft palate and bowlegged hind-limb (Fig. 2A). On radiography of fetuses, F1 fetus was observed dysplasia in third cervical vertebrae and found to only 4 of thoracic vertebrae. F2 fetus was observed only 6 of cervical vertebrae and 4 of thoracic vertebrae. Both fetuses were not shown development of thoracic, lumbar, and sacral vertebrae and also revealed caudal regression syndrome (Fig. 2B). Computed tomography was performed to carry out further examination in skeletal system. On 3-dimensional reconstruction, spina bifida which is impairment of vertebrae closure was confirmed in all vertebrae and cranial bone dysplasia was also found with non-union morphology in both fetuses. The limbs and pelvis were confirmed normal development (Fig. 2C). When ultrasonography was performed immediately after death, the brain parenchyma of fetuses was rarely observed due to severe bilateral hydrocephalus and $\mathrm{F} 2$ fetus showed more severe hydrocephalus than F1 fetus (Fig. 3A). By confirming the thoracic vertebrae with ultrasonography, it found that the spinal canal shorten by becoming narrow (Fig. 3B).

Necropsy was performed with F2 fetus. At abdomen, the developmental dysplasia in digestive system including stomach, intestine, pancreas, liver, and gall bladder was not shown (Fig. 4A). However, the liver occupied most space of abdomen by enlarging to middle abdominal cavity (Fig. 4B). The size of spleen was normal but located in more dorsal side than normal location. The position of
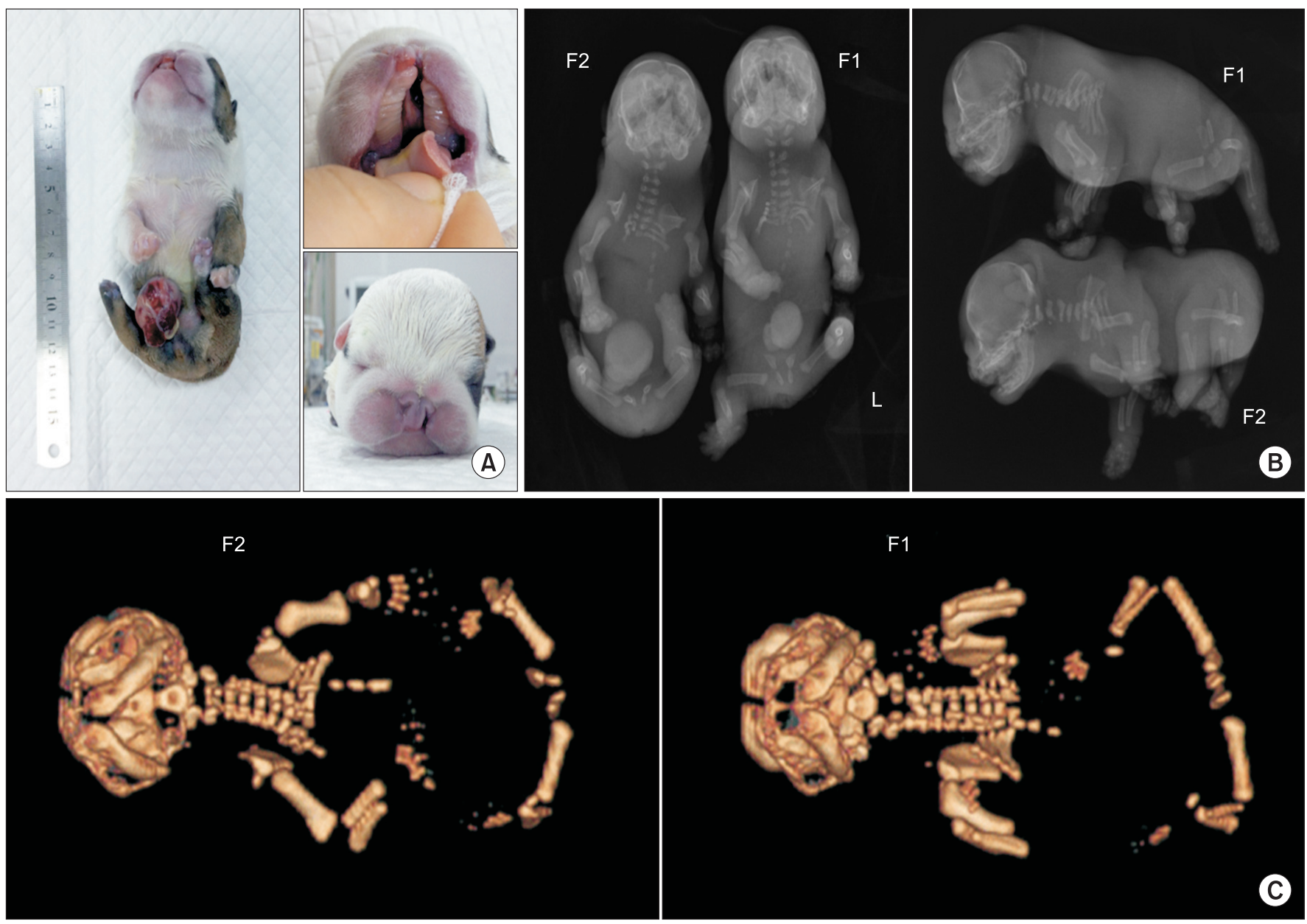

Fig. 2. Images of fetuses after $\mathrm{C}$-sec operation. (A) Gross images of fetus 2. The neonate showed cleft of lip and palate, and umbilical hernia. ( $B, C)$ Two fetuses were shown to dysplasia of vertebrae, sacrum and bowed hind-limb in both radiographs and 3-dimensional reconstruction by computed tomography. F1, fetus 1; F2, fetus 2. 

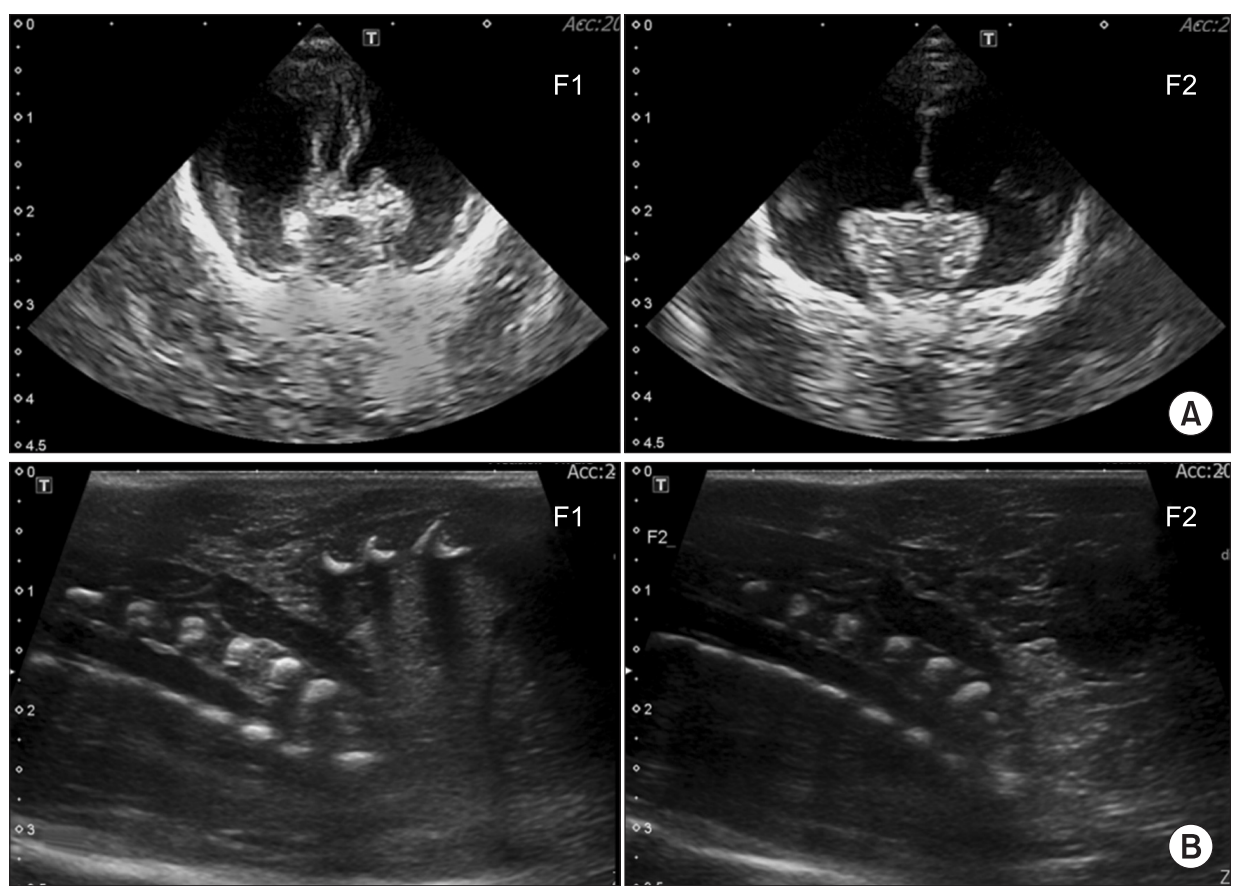

Fig. 3. Ultrasonography of brain and thoracic cavity of neonates after birth. (A) Bilateral hydrocephalus of brain parenchyma was observed in both neonates. (B) Dysplasia of spine and shortening of spinal canal was found at thoracic vertebrae in both neonates. F1, fetus 1 ; F2, fetus 2 .

kidney which shifted to caudal side was supposed to the renal ectopia and observed with abnormal morphology, not bean-shape (Fig. 4C). At the thoracic cavity, all organs including heart, lung and thymus were existed, however only three ribs and thoracic spines were existed. The lungs were remained unexpanded and the patent ductus arteriosus (PDA) was found in the heart (Fig. 4D). The skull was remained non-union and was full of cerebrospinal fluids (Fig. 4E).

\section{DISCUSSION}

The variable conditions associated with NTDs occur naturally in dogs (Bailey and Morgan, 1992). In previous studies, spina bifida has been reported to occur sporadically in breeds such as the English bulldog (Fingeroth and Smeak, 1989), Toy poodle (Parker et al., 1973), Chihuahua, mixed-bred dogs (Wilson et al., 1979), Samoyed (Furneaux et al., 1973). However, all those reports did not present a direct cause of genetic predisposition of the spina bifida. In 2013, it was first reported that the cause of NTDs is the naturally occurring mutation of the NKX28 gene through genome wide association mapping in Weimaraner dog (Safra et al., 2013).

The caudal regression syndrome is a congenital disorder associated with abnormal fetal development of the caudal part of spine (Sonek et al., 1990) and rarely reported in human. And, there are no academically reported cases of caudal regression syndrome in dogs except only one case was known through website. The male English bulldog, called Bonsai, was known to have not only the caudal regression syndrome, but also the spina bifida and swimmer's syndrome. The dog survived from 2015 to 2016, however there are no clinical report related to that case.

In dogs, it was difficult to confirm the statistics associated with birth rate of the spina bifida and caudal regression syndrome but according to human reports, the spina bifida occurred 1/2500 (Canfield et al., 2006; Kondo et al., 2009) and the caudal regression syndrome occurred $1 / 25000$ (Sonek et al., 1990). In this case, we reported an unusual case of English bulldog abnormal neonates delivered with those two severe malformations. On radiography and computed tomography, abnormal fetuses were shown non-union of cranial bone, cleft lip and palate, and umbilical hernia, specific signs of spina bifida (National Institute of Child Health and Human Development website. Neural tube defects (NTDs): Condition information. Available at: https://www.nichd.nih.gov/health/ topics/ntds /conditioninfo, 2017). And, both fetuses were not developed vertebrae under the thoracic spine and showed absence of the sacrum and bilateral bowed hind limb which are features of the most severe type caudal re- 

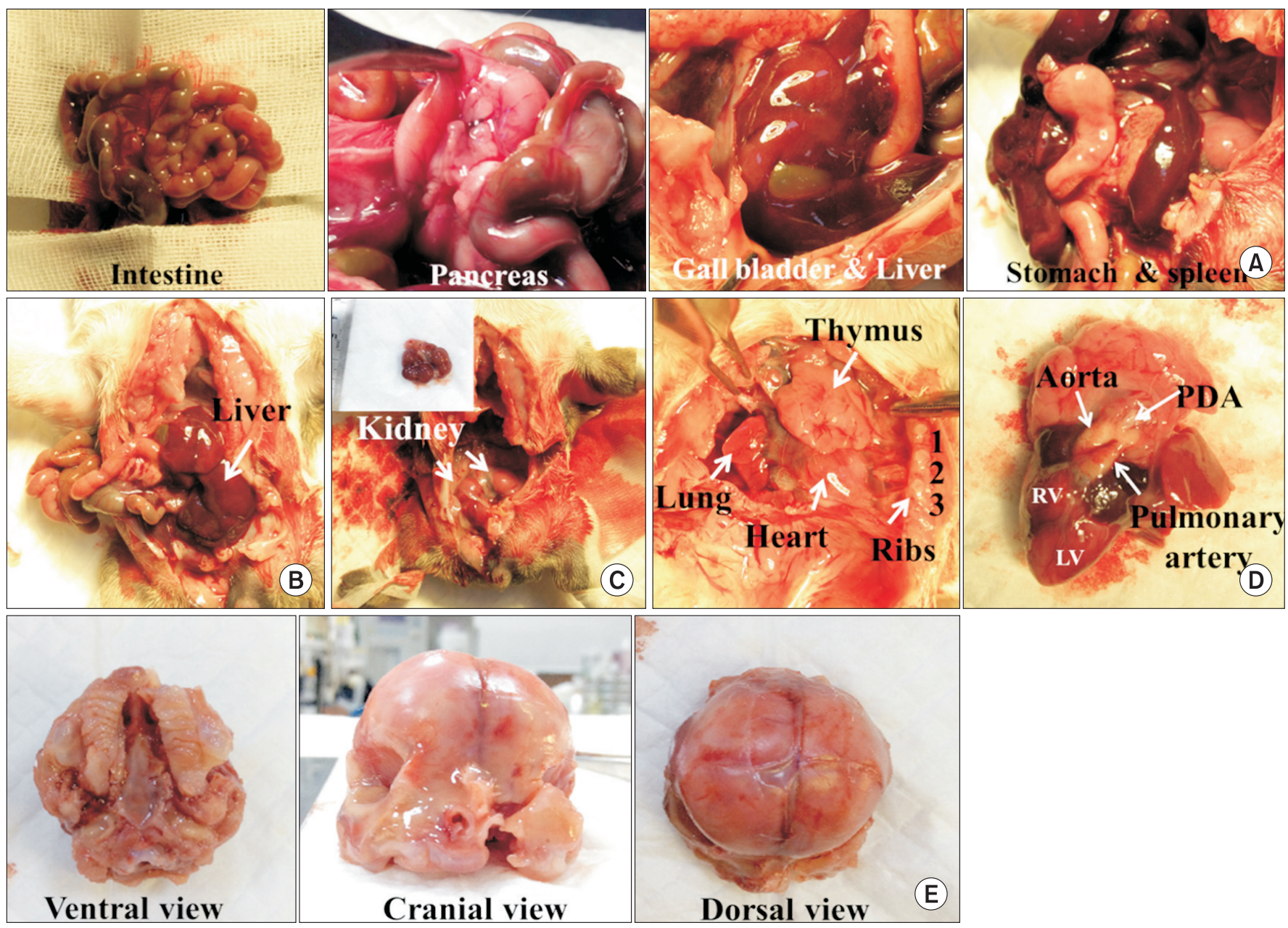

Fig. 4. Gross findings of various organs in abdomen, thoracic cavity, and brain after F2 necropsy. (A-C) Most of abdominal organs were existed (A), however enlargement of liver (B) and abnormal morphology of kidney (C) were observed. (D) All organs were existed, but PDA was observed in heart and lungs were shown to unexpanded feature. (E) Brain showed hydrocephalus feature in cranial and dorsal view. F2, fetus 2 .

gression syndrome (Sonek et al., 1990). Furthermore, the malformation of bowel and abdominal organ in caudal regression syndrome was reported in human report, liver enlargement and abnormal morphology of kidney were also founded in our case. In both fetuses, the lungs were founded to be unexpanded, which it means that fetuses could be maintained by fetal circulation in maternal body but died by dyspnea after parturition. Mother was shown to pregnancy symptoms in the first mating, but diagnosed with abortion at 4 week of gestation. In previous studies, NTDs and caudal regression syndrome were reported to occur at 3 to 4 weeks of embryo development period, therefore the first abortion is also considered to be caused by same disorder (Sonek et al., 1990; Bassuk and Kibar, 2009).
In summary, a fetus with severe multiple congenital malformation including caudal regression syndrome mostly dies in early embryo stage, and it rarely occurs that development can maintain to delivery. However, in this present case, we reported multiple congenital malformation syndrome of English bulldog that may associate with neural tube defects in embryonic stage. To understand and prevent the congenital malformation occurring in dogs, further in depth studies are needed for the breed specific genetic diversity and for the reason of behind genetic abnormality in these breeds.

\section{CONFLICTS OF INTEREST}

No potential conflict of interest relevant to this article 
was reported.

\section{ACKNOWLEDGEMENTS}

This work was supported by a grant from the National Research Foundation (NRF) of Korea, funded by the government of the Republic of Korea (grant no. NRF2018R1D1A3B07048602).

\section{ORCID}

Si-Jung Jang: https://orcid.org/0000-0003-3829-6807

Minkyung Kim: https://orcid.org/0000-0002-2135-4305

Hyeon-Jeong Lee: https://orcid.org/0000-0002-2154-239X

Young-Sung You: https://orcid.org/0000-0003-4134-3600

Jaehoon Lee: https://orcid.org/0000-0002-8340-3694

Sung-Lim Lee: https://orcid.org/0000-0002-1055-8097

\section{REFERENCES}

Aslan H, Yanik H, Celikaslan N, Yildirim G, Ceylan Y. 2001. Prenatal diagnosis of Caudal Regression Syndrome: a case report. BMC Pregnancy Childbirth. 1(1):8.

$\mathrm{Au}$ KS, Ashley-Koch A, Northrup H. 2010. Epidemiologic and genetic aspects of spina bifida and other neural tube defects. Dev Disabil Res Rev. 16(1):6-15.

Bailey CS, Morgan JP. 1992. Congenital spinal malformations. Vet Clin North Am Small Anim Pract. 22(4):985-1015.

Bassuk AG, Kibar Z. 2009. Genetic basis of neural tube defects. Semin Pediatr Neurol.16(3):101-110.

Canfield MA, Honein MA, Yuskiv N, Xing J, Mai CT, Collins JS, Devine O, Petrini J, Ramadhani TA, Hobbs CA, Kirby RS. 2006. National estimates and race/ethnic-specific variation of selected birth defects in the United States, 1999-2001. Birth Defects Res A Clin Mol Teratol. 76(11):747-756.

Fingeroth JM, Smeak DD. 1989. Laminotomy of the axis for surgical access to the cervical spinal cord. A case report. Vet Surg. 18(2):123-129.

Furneaux RW, Doige CE, Kaye MM. 1973. Syringomyelia and spina bifida occulta in a Samoyed dog. Can Vet J. 14(12):317321.

Harris MJ, Juriloff DM. 2010. An update to the list of mouse mutants with neural tube closure defects and advances toward a complete genetic perspective of neural tube closure. Birth Defects Res A Clin Mol Teratol. 88(8):653-669.

Harris MJ. 2009. Insights into prevention of human neural tube defects by folic acid arising from consideration of mouse mutants. Birth Defects Res A Clin Mol Teratol. 85(4):331339.

Hertzler DA 2nd, DePowell JJ, Stevenson CB, Mangano FT. 2010. Tethered cord syndrome: a review of the literature from embryology to adult presentation. Neurosurg Focus. 29(1):E1.

Kondo A, Kamihira O, Ozawa H. 2009. Neural tube defects: prevalence, etiology and prevention. Int J Urol. 16(1):49-57.

National Institute of Child Health and Human Development website. 2017. Neural tube defects (NTDs): Condition information. Available at: https://www.nichd.nih.gov/health/ topics/ntds /conditioninfo. Retrieved Nov 30.

Parker AJ, Park RD, Byerly CS, Stowater JL. 1973. Spina bifida with protrusion of spinal cord tissue in a dog. J Am Vet Med Assoc. 163(2):158-160.

Safra N, Bassuk AG, Ferguson PJ, Aguilar M, Coulson RL, Thomas N, Hitchens PL, Dickinson PJ, Vernau KM, Wolf ZT, Bannasch DL. 2013. Genome-wide association mapping in dogs enables identification of the homeobox gene, NKX2-8, as a genetic component of neural tube defects in humans. PLoS Genet. 9(7):e1003646.

Sen KK, Patel M. 2007. Caudal regression syndrome. Med J Armed Forces India. 63(2):178-179.

Sonek JD, Gabbe SG, Landon MB, Stempel LE, Foley MR, Shubert-Moell K. 1990. Antenatal diagnosis of sacral agenesis syndrome in a pregnancy complicated by diabetes mellitus. Am J Obstet Gynecol. 162(3):806-808.

Wilson JW, Kurtz HJ, Leipold HW, Lees GE. 1979. Spina bifida in the dog. Vet Pathol. 16(2):165-179. 